\title{
miR-375 inhibits cell growth and correlates with clinical outcomes in tongue squamous cell carcinoma
}

\author{
LINGFEI JIA ${ }^{1,2^{*}}$, YIPING HUANG ${ }^{3 *}$, YUNFEI ZHENG $^{1 *}$, MINGYUE LYU $^{1}$, CHUNAN ZHANG $^{1}$, \\ ZHEN MENG $^{1,2}$, YEHUA GAN ${ }^{1,2}$ and GUANGYAN YU ${ }^{1}$ \\ ${ }^{1}$ Department of Oral and Maxillofacial Surgery, ${ }^{2}$ Central Laboratory and ${ }^{3}$ Department of Orthodontics, \\ Peking University School and Hospital of Stomatology, Beijing 100081, P.R. China
}

Received September 17, 2014; Accepted December 10, 2014

DOI: $10.3892 /$ or.2015.3759

\begin{abstract}
R-375 has been implicated in various types of cancers. However, its role in tongue squamous cell carcinoma (TSCC) remains unclear. This study aimed to investigate the effects of miR-375 on cell growth and the prognosis of TSCC patients. Using quantitative reverse transcription-polymerase chain reaction, we evaluated miR-375 expression in TSCC samples and TSCC cell lines. The results showed that miR-375 expression was significantly reduced in the TSCC tissues and cell lines. A low level expression of miR-375 in TSCC patients was related to poor of prognosis. Moreover, the effects of miR-375 overexpression on cell proliferation, the cell cycle and the expression of Sp1 and cyclin D1 were examined in TSCC cells. We demonstrated that overexpression of miR-375 significantly inhibited the cell proliferation and cell cycle progression in TSCC cell lines. Overexpression of miR-375 inhibited Sp1 expression by targeting the 3' untranslated region of the Sp1 transcript. The knockdown of Sp1 expression resulted in the subsequent downregulation of cyclin D1. Taken together, our study suggests that miR-375 inhibits the cell growth, and its expression is correlated with clinical outcomes in TSCC.
\end{abstract}

Correspondence to: Professor Yehua Gan, Laboratory of Molecular Biology, Peking University School and Hospital of Stomatology, 22 Zhongguancun Avenue South, Haidian, Beijing 100081, P.R. China

E-mail: kqyehuagan@bjmu.edu.cn

Professor Guangyan Yu, Department of Oral and Maxillofacial Surgery, Peking University School and Hospital of Stomatology, 22 Zhongguancun Avenue South, Haidian, Beijing 100081, P.R. China E-mail: gyyu@263.net

\section{${ }^{*}$ Contributed equally}

Key words: oral cancer, tongue squamous cell carcinoma, miRNA, miR-375, Sp1, cyclin D1

\section{Introduction}

Squamous cell carcinoma of the oral cavity and oropharynx is the sixth most frequent solid cancer worldwide (1). Tongue squamous cell carcinoma (TSCC) is the most common types of oral carcinoma and is well-known for its rapid proliferation (2). Prognostic assessment is critical for making better therapeutic choices for patients, and the tumor-node-metastasis (TNM) staging system is the key prognostic determinant for TSCC patients in clinical practice (3). However, conventional prognostic factors based on clinicopathological features remain inadequate, and are unable to discriminate tumors at the same clinical stage but with distinct clinical outcomes. The lack of efficient diagnostic and prognostic biomarkers is responsible for the high mortality rates (4). Therefore, it is necessary to identify biomarkers that can provide additional prognostic information beyond the standard clinical prognostic system for TSCC patients (5).

microRNAs (miRNAs) are a group of endogenously expressed small non-coding RNAs involved in the regulation of gene expression at the post-transcriptional level (6). They show specific expression patterns in cancers, which enable them to be biomarkers for cancer risk and prognosis (7). Subsets of miRNAs, including miR-195 (8), miR-21 (9), miR-200b and miR-15b (10), have been identified as potential diagnostic and prognostic markers in TSCC patients. Other tumor-related miRNAs may also have value for the prediction of prognosis in TSCC patients.

Recent studies have shown that the expression of miR-375 is associated with various clinicopathological parameters in oral squamous cell carcinoma (OSCC) (11), and is correlated with clinical outcomes in head and neck squamous cell carcinoma (HNSCC) (12). However, these studies included heterogeneous groups of patients with cancers from different subsites of the head and neck, including tongue, gingival, buccal, lip, larynx and oropharynx carcinomas. Since the gene expression patterns in HNSCC at different subsites may not be equally associated with cancer prognosis $(13,14)$, a study focusing on a specific anatomical subsite, such as the anterior body of the tongue, is likely to provide more accurate and clinically useful information on the prognostic significance of miR-375.

miR-375 frequently shows reduced expression in various cancers, and it may act as a tumor suppressor by targeting 
JAK-2 (15), PDK-1 (16), IGF1R (17) and ASCL-1 (18). However, the role of miR-375 and the underlying mechanism in TSCC remain to be explored. Sp1 is a target gene of miR-375 in cervical squamous cell cancer (19). Moreover, Sp1 upregulates cyclin D1 expression by binding to the cyclin D1 promoter (20) and is required for cell cycle progression through the $G_{1}$ phase (21). Thus, it was reasonable to test whether miR-375 directly targets Sp1 and subsequently downregulates cyclin D1 to induce cell cycle arrest in TSCC.

In the present study, we examined the expression of miR-375 in 105 pairs of TSCC samples and matched adjacent normal tissues, and the association of miR-375 expression with the overall survival of TSCC patients. Moreover, we tested whether miR-375 downregulates Sp1 expression by targeting the Sp1 transcript and subsequently downregulates cyclin D1 to induce cell cycle arrest in TSCC cells. Our results suggest important roles for miR-375 in TSCC pathogenesis and support its potential application in the evaluation of patient prognosis.

\section{Materials and methods}

Clinical specimens. Paired primary TSCC samples and adjacent histologically normal tissues were obtained from 105 patients who were admitted to the Department of Oral and Maxillofacial Surgery of the Peking University School of Stomatology (Beijing, China) between 2008 and 2011. None of the patients received treatment prior to radical surgical treatment. Tumor tissues and matched nonmalignant tissues, at least $1.5 \mathrm{~cm}$ distal to the tumor margins, were snap-frozen in liquid nitrogen and then stored at $-80^{\circ} \mathrm{C}$ until use. None of the TSCC patients had received adjuvant chemotherapy or radiotherapy before surgery. The clinicopathological characteristics of the patients are summarized in Table I. This study was approved by the Ethics Committee of the Peking University School of Stomatology, and all samples were obtained from patients who had signed informed consent forms.

Cell lines and culture. Primary normal human oral keratinocyte (HOK) cells were purchased and cultured in a keratinocyte growth medium (ScienCell Research Laboratories, San Diego, CA, USA) according to the manufacturer's instructions. Human TSCC cell lines, SCC-15 and CAL-27, were obtained from the American Type Culture Collection (Manassas, VA, USA) and cultured in Dulbecco's modified Eagle's medium supplemented with $10 \%$ fetal bovine serum and $1 \%$ antibiotics at $37^{\circ} \mathrm{C}$ in a humidified atmosphere of $5 \% \mathrm{CO}_{2}$ and $95 \%$ air.

Vector construction. A 417-base pair (bp) fragment of the $3^{\prime}$ untranslated region (UTR) of the human Sp1 transcript containing the miR-375 binding site (19) was amplified by polymerase chain reaction (PCR). It was then cloned into a modified version of pcDNA3.1(+) containing a firefly luciferase reporter gene (a gift from Brigid L.M. Hogan, Duke University, Durham, NC, USA) (22) and named wild-type Sp1 3'UTR. The following primers were used to clone the wildtype Sp1 3'UTR: sense, 5'-GAA TTC TTT TCC TTG TAT GTT CTT GGG T-3' and antisense, 5'-CTC GAG GCA GGT CTC TTT TAA TAT TGG C-3'. Site-directed mutagenesis of the miR-375 binding site in the Sp1 3'UTR was performed using a Site-Directed Mutagenesis Kit (SBS Genetech,
Table I. Relationship between expression of miR-375 and the clinicopathological factors in the 105 TSCC patients.

\begin{tabular}{|c|c|c|c|}
\hline Characteristics & No. & $\begin{array}{c}\operatorname{miR}-375(\mathrm{~T} / \mathrm{N}) \\
(\mathrm{mean} \pm \mathrm{SD})\end{array}$ & P-value \\
\hline Gender & & & 0.132 \\
\hline Male & 49 & $0.194 \pm 0.245$ & \\
\hline Female & 56 & $0.131 \pm 0.169$ & \\
\hline Age (years) & & & 0.424 \\
\hline$<60$ & 65 & $0.172 \pm 0.243$ & \\
\hline$\geq 60$ & 40 & $0.142 \pm 0.138$ & \\
\hline Tumor size & & & 0.041 \\
\hline $\mathrm{T}_{1}-\mathrm{T}_{2}$ & 67 & $0.189 \pm 0.232$ & \\
\hline $\mathrm{T}_{3}-\mathrm{T}_{4}$ & 38 & $0.111 \pm 0.153$ & \\
\hline Differentiation & & & 0.896 \\
\hline Well & 45 & $0.168 \pm 0.257$ & \\
\hline Moderate & 47 & $0.159 \pm 0.178$ & \\
\hline Poor & 13 & $0.137 \pm 0.122$ & \\
\hline Clinical stage & & & 0.419 \\
\hline I-II & 59 & $0.175 \pm 0.211$ & \\
\hline III-IV & 46 & $0.142 \pm 0.208$ & \\
\hline Node metastasis & & & 0.266 \\
\hline No & 62 & $0.178 \pm 0.247$ & \\
\hline Yes & 43 & $0.135 \pm 0.136$ & \\
\hline Status & & & 0.028 \\
\hline Surviving & 60 & $0.197 \pm 0.235$ & \\
\hline Deceased & 45 & $0.111 \pm 0.158$ & \\
\hline
\end{tabular}

$\mathrm{T}$, tumor; $\mathrm{N}$, nonmalignant tissue; $\mathrm{T} 1-\mathrm{T} 4, \mathrm{~T}$ stage of the TNM classification system.

Beijing, China). The product was named mutant Sp1 3'UTR. The following primers were used to clone the mutant $\mathrm{Sp} 1$ 3'UTR: sense, 5'-GAA TGA TAG CCC AGT TGT TAA AGA AAT CTT GT-3' and antisense, 5'-ACA AGA TTT CTT TAA CAA CTG GGC TAT CAT TC-3'. A 1,934-bp fragment of human cyclin D1 promoter (-1,696-bp to +238 -bp relative to the transcription start site) was amplified from the genomic DNA of HeLa cells and cloned into the PGL3-Enhancer vector (Promega, Madison, WI, USA). The primers used to clone the cyclin D1 promoter were as follows: sense, $5^{\prime}-\mathrm{GCG}$ GTA CCG CTA GCC AGC TGG GCC GCC CTT GT-3' and antisense, 5'-ATC CAT GGA AGC TTT GGG GCT CTT CCT GGG CA-3'. All constructs were confirmed by DNA sequencing.

RNA oligoribonucleotide. A chemically modified doublestranded miR-375 mimic and the corresponding miRNA mimic control were designed and purchased from RiboBio Co. (Guangzhou, China). A small interfering RNA (siRNA) targeting the human Sp1 transcript (siSp1) and the corresponding scrambled control were purchased from Integrated Biotech Solutions Co. (Shanghai, China). The miRNA and siRNA sequences are listed in Table II. 
Table II. Sequences of RNA and DNA oligonucleotides.

\begin{tabular}{lll}
\hline Name & \multicolumn{1}{c}{ Sense strand/sense primer (5'-3') } & \multicolumn{1}{c}{ Antisense strand/antisense primer (5'-3') } \\
\hline $\begin{array}{l}\text { Primers for qRT-PCR } \\
\text { miR-375 RT primer }\end{array}$ & $\begin{array}{l}\text { GTCGTATCCAGTGCAGGGTCCGAGGTAT } \\
\text { TCGCACTGGATACGACTCACGC }\end{array}$ & \\
miR-375 & GTGCAGGGTCCGAGGT & AGCCGTTTGTTCGTTCGGCT \\
U6 & CTCGCTTCGGCAGCACA & AACGCTTCACGAATTTGCGT \\
Sp1 & ACCAGAATAAGAAGGGAGG & GGTGGTAATAAGGGCTGAA \\
Cyclin D1 & GTGCTGCGAAGTGGAAACC & ATCCAGGTGGCGACGATCT \\
$\begin{array}{l}\text { B-actin } \\
\text { miRNA mimic }\end{array}$ & CGG GAAATC GTG CGT GAC & CAG GCA GCT CGT AGC TCT T \\
miR-375 mimic & UUUGUUCGUUCGGCUCGCGUGA & AAACAAGCAAGCCGAGCGCACU \\
miRNA mimic control & UUUGUACUACACAAAAGUACUG & AAACAUGAUGUGUUUCAUGAC \\
siRNA duplexes & & \\
Sp1 siRNA & UGUAGAGUCUGCCAACUGACCUGUCTT & GACAGGUCAGUUGGCAGACUCUACATT \\
siRNA control (scramble) & UUC UCC GAACGU GUCACG UTT & ACG UGA CAC GUU CGG AGA ATT \\
\hline
\end{tabular}

Transient transfection. Cells were plated into 6-well plates before transfection. After reaching $80 \%$ confluency, the cells were transfected with $100 \mathrm{nM}$ miRNA mimic or siRNA using Lipofectamine 2000 (Invitrogen, Carlsbad, CA, USA) according to the manufacturer's procedure. The cells were harvested $48 \mathrm{~h}$ after transfection.

RNA isolation and quantitative reverse-transcription $(q R T)-P C R$. Total RNA was extracted using the TRIzol reagent (Invitrogen) according to the manufacturer's procedure, and then reverse-transcribed into complementary DNA (cDNA) using a cDNA reverse transcription kit (Applied Biosystems, Foster City, CA, USA). Quantitative PCR was conducted with the ABI Prism 7500 real-time PCR system (Applied Biosystems). The following thermal settings were used: $95^{\circ} \mathrm{C}$ for $10 \mathrm{~min}$ followed by 40 cycles of $95^{\circ} \mathrm{C}$ for $15 \mathrm{sec}$ and $60^{\circ} \mathrm{C}$ for $1 \mathrm{~min}$. The primers used for miR-375, Sp1, cyclin D1, U6 (internal control for miRNAs) and $\beta$-actin (internal control for mRNAs and lncRNAs) are listed in Table II. The data was analyzed using the $2^{-\Delta \Delta \mathrm{Ct}}$ relative expression quantity as described previously (8).

Cell proliferation assays. Cell proliferation was determined using the Cell Counting Kit-8 (CCK-8, Dojindo, Kumamoto, Japan). SCC-15 and CAL27 cells were plated into 96-well plates $\left(2 \times 10^{3}\right.$ cells/well). After transfection with miR-375 mimic or the scrambled control, CCK-8 (10 $\mu \mathrm{l})$ was added to each well at 24,48 , and $72 \mathrm{~h}$, and the plates were incubated at $37^{\circ} \mathrm{C}$ for $3 \mathrm{~h}$. The absorbance at $450 \mathrm{~nm}$ was measured using a microplate spectrophotometer (Bio-Tek Instruments).

Colony formation assay. At $24 \mathrm{~h}$ after transfection, the cells were plated into $60-\mathrm{mm}$ dishes at an initial density of 500 cells/dish and cultured until colonies were visible (10 days). Cell colonies were fixed with cold methanol for $20 \mathrm{~min}$ and stained with $0.25 \%$ crystal violet for $30 \mathrm{~min}$. The number of cells that formed a clone was calculated.
Cell cycle analysis. At $48 \mathrm{~h}$ post-transfection, CAL27 cells were harvested by trypsinization and washed with phosphate-buffered saline. The cell cycle was analyzed with fluorescence-activated cell sorting (FACS) using FACSCalibur flow cytometry (Becton Dickinson, Bedford, MA, USA) as described previously (8).

Dual luciferase reporter assay. Luciferase assays were performed as described previously (23). Briefly, SCC-15 and CAL27 cells grown in a 48-well plate were cotransfected with the miR-375 mimic or the miRNA mimic control $(100 \mathrm{nM})$, the luciferase reporter plasmid (40 ng/well) and pRL-TK, a plasmid expressing Renilla luciferase (4 ng/well; Promega). Luciferase activity was measured $24 \mathrm{~h}$ after transfection using the Dual-luciferase reporter assay system (Promega).

Western blot analysis. Western blotting was performed as described previously (8). Primary antibodies against Sp1 (Cell Signaling Technology, Beverly, MA, USA), cyclin D1 and $\beta$-actin (both from Santa Cruz Biotechnology, Santa Cruz, CA, USA) were diluted at 1:1,000. The intensities of the bands obtained by western blotting were quantified using ImageJ software (http://rsb.info.nih.gov/ij). The background was subtracted, and the signal of the target bands was normalized to that of the $\beta$-actin band.

Statistical analysis. Statistical analyses were performed using SPSS for Windows ver. 16.0 (IBM, Armonk, NY, USA). All data are expressed as the mean \pm standard deviation (SD). Differences between groups were analyzed by the Student's t-test. A one-way ANOVA was used to analyze the relationship between miR-375 expression and the clinicopathological characteristics. Survival curves were constructed by the Kaplan-Meier method and were compared using the log-rank test. The Cox regression model was applied to simultaneously adjust all potential prognostic variables. Experiments with cell cultures were conducted at least in triplicate. A two-tailed 
A

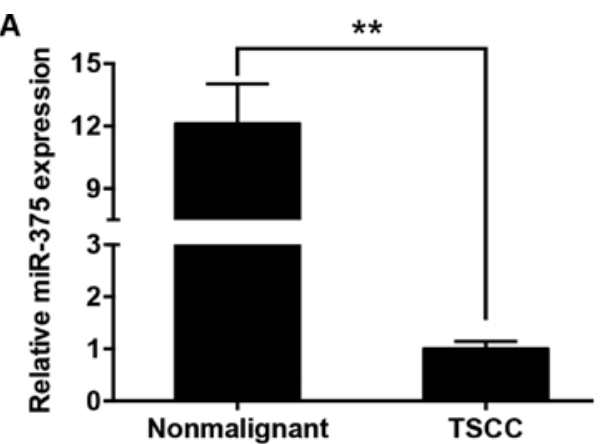

B

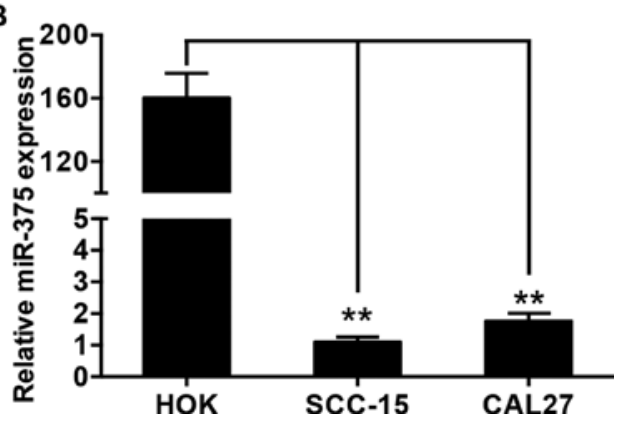

Figure 1. miR-375 expression is reduced in the TSCC clinical specimens and cell lines. (A) miR-375 expression was reduced in the human TSCC tissues compared with that in the matched adjacent nonmalignant tissues. (B) miR375 expression was reduced in the SCC-15 and CAL27 cells compared with that in the primary normal human oral keratinocyte (HOK) cells. Data are shown as the mean $\pm \mathrm{SD}\left({ }^{* *} \mathrm{P}<0.01\right)$.

value of $\mathrm{P}<0.05$ was considered to indicate a statistically significant difference.

\section{Results}

miR-375 expression is reduced in TSCC and is correlated with cancer progression. The expression of miR-375 was examined in 105 pairs of tongue cancer tissues and matched adjacent histologically normal tissues by qRT-PCR. The average expression level of miR-375 was significantly decreased in the tumor tissues when compared with the level in the normal tissues (Fig. 1A). Moreover, miR-375 expression was substantially reduced in the TSCC cell lines, SCC-15 and CAL27, compared with that in the HOK cells (Fig. 1B). The relationship between miR-375 expression and the clinicopathological factors of TSCC are presented in Table I. By normalizing the miR-375 expression levels in the tumor tissues to those in the adjacent nonmalignant tissues (Tumor/Nonmalignant, T/N), we observed that miR-375 expression $(\mathrm{T} / \mathrm{N})$ was significantly correlated with tumor size $(\mathrm{P}=0.041)$ and patient mortality $(\mathrm{P}=0.028)$.

Decreased miR-375 expression is associated with poor overall survival in the TSCC patients. With the mean fold change of miR-375 expression $(\mathrm{T} / \mathrm{N})$ chosen as the cut-off point, the patients were divided into high and low expression groups. The patients with high miR-375 expression survived significantly longer than those with low miR-375 expression (Fig. 2). Moreover, we conducted multivariable Cox proportional hazards analysis to identify whether miR-375 is an independent prognostic covariate for TSCC patients. Our results showed that low miR-375 expression in TSCC was associated with a poor prognosis in terms of overall survival $(\mathrm{P}=0.044$,

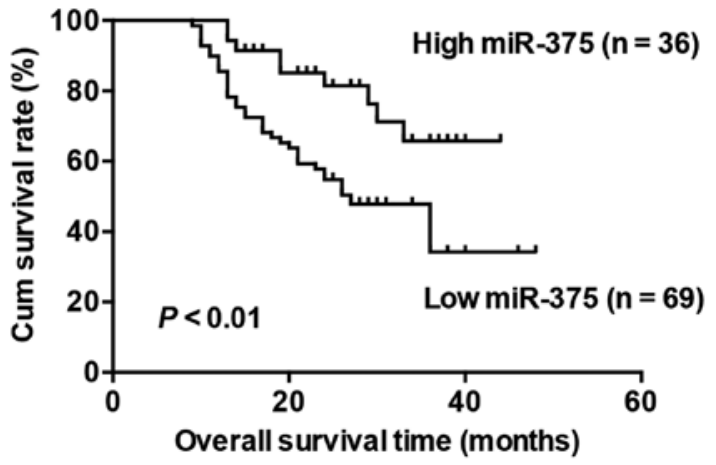

Figure 2. Decreased expression of miR-375 is correlated with poor survival in TSCC patients. The mean miR-375 expression level $(\mathrm{T} / \mathrm{N}=0.160)$ in the tumor samples was chosen as the cut-off point. Kaplan-Meier curves with log-rank tests showed that patients with high miR-375 expression (T/N-fold change $>0.160)$ survived significantly longer $(\mathrm{P}=0.044)$ than patients with low miR-375 expression $(\mathrm{T} / \mathrm{N}$-fold change $<0.160)$.

relative risk $=0.449$ ), independent of the other clinical covariates (Table III).

Overexpression of miR-375 inhibits cell proliferation and cell cycle progression in TSCC cell lines. To investigate the biological function of miR-375 in TSCC, we transfected the miR-375 mimic into TSCC cells, and confirmed that miR-375 expression was significantly increased in the SCC-15 and CAL27 cells at $48 \mathrm{~h}$ post-transfection (data not shown). CCK- 8 and colony formation assays showed that overexpression of miR-375 significantly inhibited the proliferation of the SCC-15 and CAL27 cells (Fig. 3A and B), and resulted in substantial accumulation of cells in the $\mathrm{G}_{1}$-phase (Fig. 3C).

miR-375 inhibits Spl expression by targeting its 3'UTR. According to a previous study, the human Sp1 transcript contains a putative miR-375 target site in its 3'UTR (19) (Fig. 4A). We constructed a luciferase reporter plasmid containing the human Sp1 3'UTR with the miR-375 target site intact (wild-type) or mutated. SCC-15 and CAL27 cells were co-transfected with the miR-375 mimic, the miRNA mimic control, and the reporter plasmid. Ectopic expression of miR-375 significantly suppressed the luciferase activity of the reporter with the wild-type Sp1 3'UTR, but not that of the reporter with the mutant Sp1 3'UTR, at 24 h post-transfection (Fig. 4B). Meanwhile, overexpression of miR-375 significantly decreased the mRNA and protein levels of endogenous Sp1 at $48 \mathrm{~h}$ post-transfection compared to the negative control (Fig. 4C and D).

Overexpression of miR-375 downregulates cyclin D1 expression by targeting Sp1. To explore whether miR-375 also regulates the expression of cyclin D1, we measured its mRNA and protein levels in SCC-15 and CAL27 cells transfected with the miR-375 mimic and the miRNA mimic control. Overexpression of miR-375 significantly downregulated the mRNA and protein levels of cyclin D1 (Fig. 5A and B). A previous study reported that Sp1 binding sites in the cyclin D1 promoter are involved in transcriptional activation of the gene (20). We constructed a luciferase reporter plasmid containing 

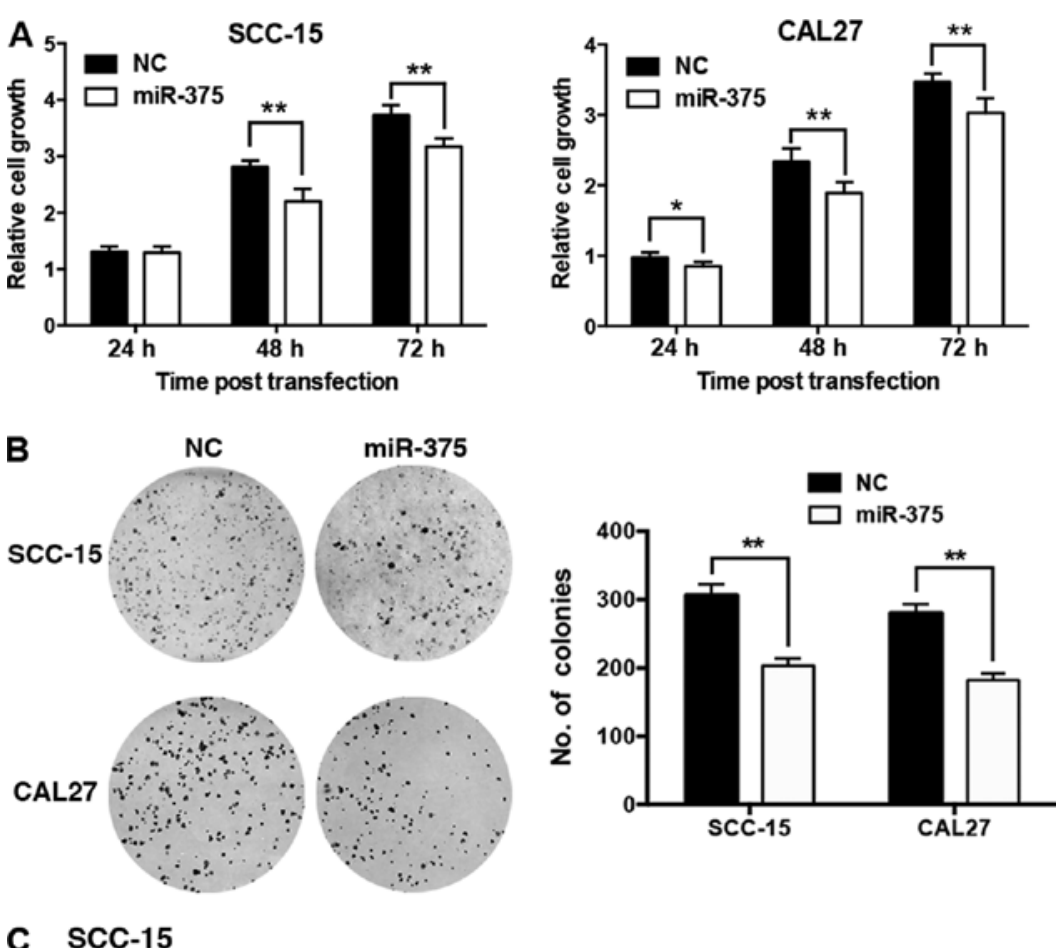

C SCC-15
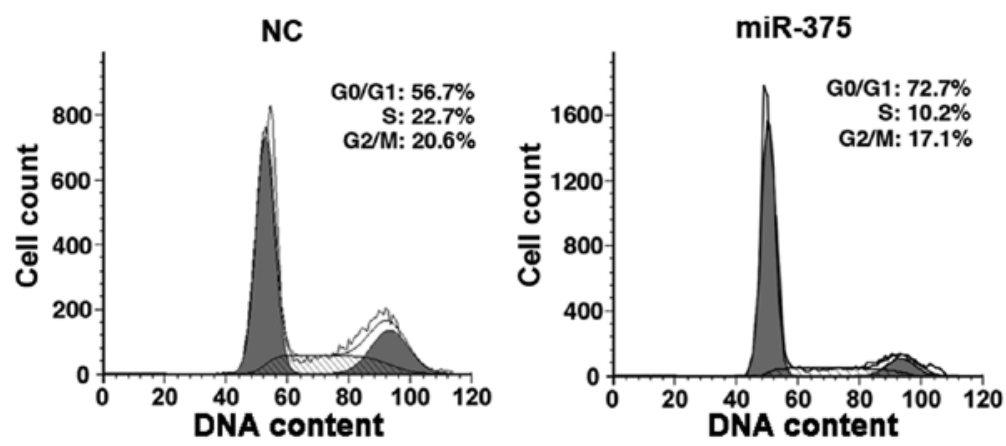

CAL27

NC

$\operatorname{miR}-375$
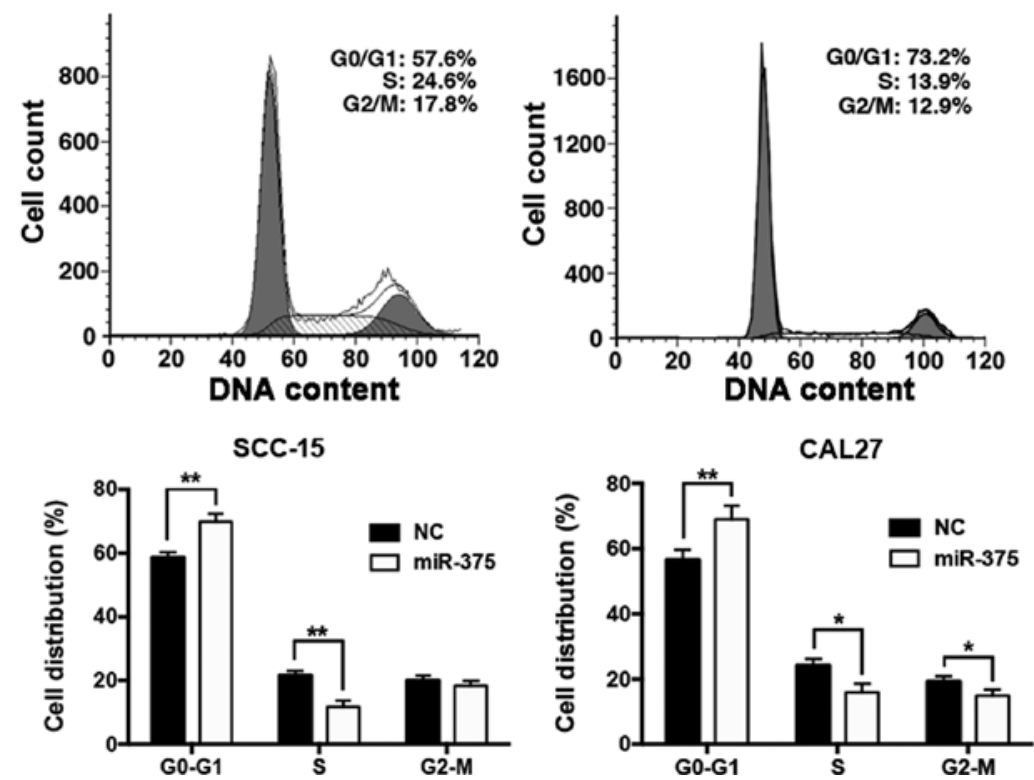

Figure 3. Overexpression of miR-375 inhibits proliferation, colony formation, and cell cycle progression. (A) Inhibition of cell proliferation by overexpression of miR-375. SCC-15 and CAL27 cells were transfected with the miR-375 mimic or the miRNA mimic control (negative control, NC). Proliferation was measured using CCK-8 assays. (B) Inhibition of colony formation by overexpression of miR-375. Representative results of colony formation by the SCC-15 and CAL27 cells transfected as in A are shown. (C) Inhibition of cell cycle progression by overexpression of miR-375. SCC-15 and CAL27 cells were transfected as in A. Cells were stained with propidium iodide (PI) at $48 \mathrm{~h}$ post-transfection and analyzed by FACS. Data are shown as the mean $\pm \mathrm{SD}\left({ }^{*} \mathrm{P}<0.05,{ }^{* *} \mathrm{P}<0.01\right)$. 
Table III. Multivariate analysis of various prognostic variables in the TSCC patients using Cox's regression analysis.

\begin{tabular}{|c|c|c|c|c|c|}
\hline Variables & No. of cases & P-value & Regression coefficient & Relative risk & $95 \% \mathrm{CI}$ \\
\hline Tumor size & & 0.177 & 0.322 & 1.545 & $0.822-2.906$ \\
\hline $\mathrm{T}_{1}-\mathrm{T}_{2}$ & 67 & & & & \\
\hline $\mathrm{T}_{3}-\mathrm{T}_{4}$ & 38 & & & & \\
\hline Differentiation & & 0.723 & 0.307 & 1.115 & $0.611-2.035$ \\
\hline Well & 45 & & & & \\
\hline Moderate and poor & 60 & & & & \\
\hline Clinical stage & & 0.259 & 0.341 & 1.469 & $0.754-2.865$ \\
\hline I-II & 59 & & & & \\
\hline III-IV & 46 & & & & \\
\hline Node metastasis & & 0.212 & 0.334 & 1.517 & $0.789-2.919$ \\
\hline No & 62 & & & & \\
\hline Yes & 43 & & & & \\
\hline miR-375 expression & & 0.044 & 0.396 & 0.449 & $0.207-0.978$ \\
\hline High & 36 & & & & \\
\hline Low & 69 & & & & \\
\hline
\end{tabular}

CI, confidence interval.

the cyclin D1 promoter with the Sp1 binding sites. When SCC-15 or CAL27 cells were co-transfected by the miR-375 mimic and the cyclin D1 promoter luciferase reporter plasmid, the luciferase activity level was significantly reduced compared with the control (Fig. 5C). Moreover, luciferase activity was markedly suppressed by the introduction of siRNA against the Sp1 transcript into the SCC-15 and CAL27 cells, which mimicked the effects of miR-375 overexpression (Fig. 5D). In addition, the transfection of Sp1 siRNA in the SCC-15 or CAL27 cells significantly reduced the protein expression levels of both Sp1 and cyclin D1, as expected (Fig. 5E).

Knockdown of Sp1 inhibits cell proliferation and cell cycle progression in TSCC cell lines. To test whether the downregulation of Sp1 is involved in the antitumor actions of miR-375, we knocked down Sp1 expression in the SCC-15 and CAL27 cells. The knockdown of Sp1 in the TSCC cells suppressed cell proliferation (Fig. 6A) and colony formation (Fig. 6B), and induced a $\mathrm{G}_{1}$ phase arrest (Fig. 6C).

\section{Discussion}

In the present study, we demonstrated that miR-375 expression was reduced in TSCC specimens and cell lines, and was correlated with tumor size and patient mortality. Moreover, our results suggest that low expression of miR-375 was correlated with shorter survival of TSCC patients, and served as a prognostic factor independent of other clinicopathological factors. Similarly, a study of 123 HNSCC patients, including patients with cancers of the oral cavity, oropharynx, and larynx, showed that a low level of miR-375 was a potential prognostic marker of poor outcome in patients. However, this study found no significant correlation between miR-375 and any of the clinicopathological parameters studied (12). By contrast, a recent study of 26 OSCC patients, including patients with cancers of the tongue, gingival and buccal tissue, revealed that miR-375 expression was reduced and was correlated with clinical stage and tumor size (11). However, the prognostic implication of miR-375 in oral carcinoma was not analyzed. Variation in correlation with clinicopathological parameters and the prognostic significance of miR-375 in previous studies may be attributable to differences in sample size and the inclusion of tumors from different subsites of the head and neck. Thus, our study of 105 patients with HNSCC at a single anatomical site, the anterior tongue, is valuable for confirming the prognostic value of miR-375 in TSCC patients.

Previous reports have shown that low miR-375 expression is also related to poor survival and a poor therapeutic outcome in gastric cancer (24), esophageal squamous cell carcinoma $(17,25)$, non-small cell lung cancer $(26,27)$ and glioma (28). Thus, as a tumor suppressor, miR-375 seems to be a useful prognostic factor for various types of malignant tumors. However, upregulation of miR-375 was reported to correlate with a poor prognosis in pediatric acute myeloid leukemia (29). Variation in the prognostic significance of miR-375 in previous studies may be attributable to differences in cancer type.

The present study also demonstrated that overexpression of miR-375 in TSCC cell lines inhibited cell proliferation and cell cycle progression, consistent with previous studies of avian leucosis (30) and colorectal cancer (31). We focused on the biological effects of miR-375 on TSCC growth since miR-375 was significantly correlated with tumor size but showed no relationship with other clinicopathological parameters. Furthermore, we confirmed that $\mathrm{Sp} 1$ is a direct target gene of miR-375, consistent with a previous study of squamous 
A

miR-375 target site in Sp1 3'UTR

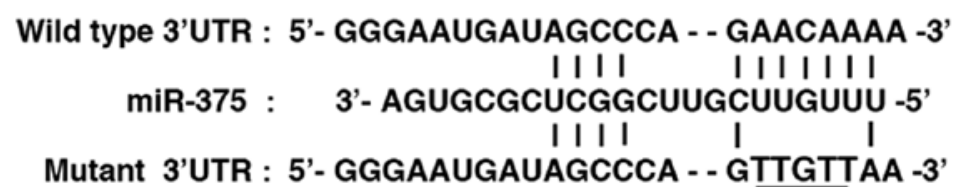

B

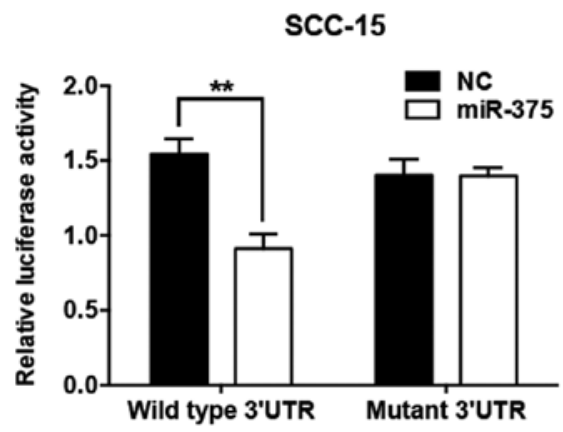

C

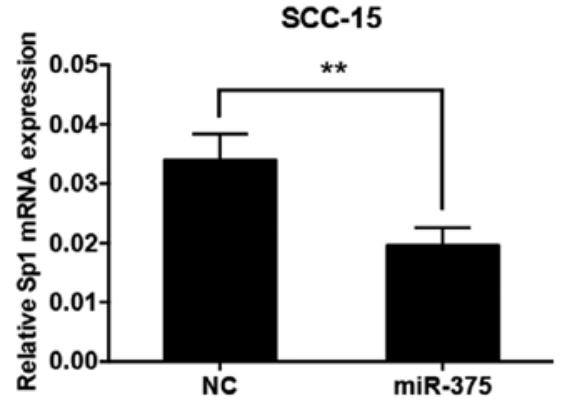

D

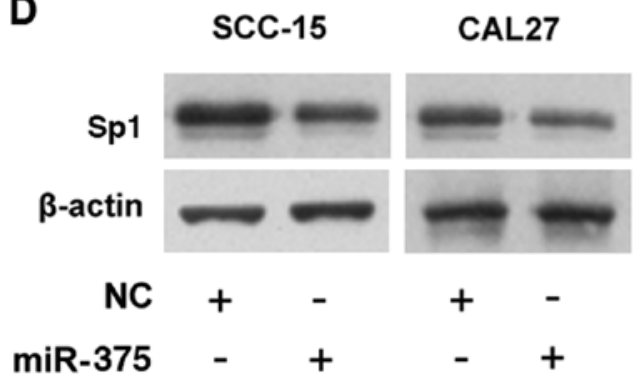

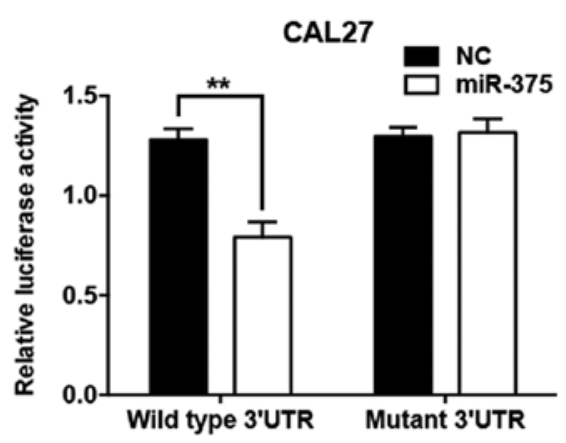
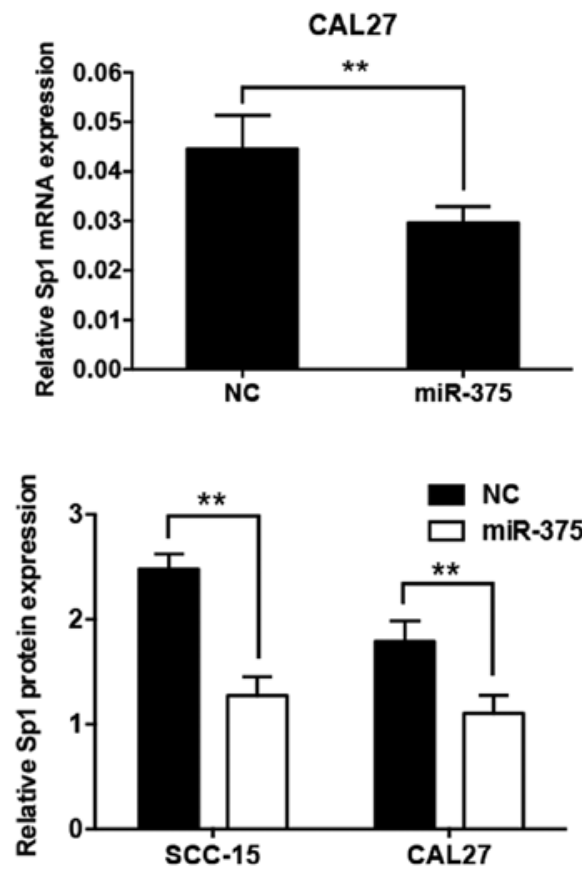

Figure 4. Sp1 is a direct target gene of miR-375. (A) The seed sequence of miR-375 (middle) matches the 3'UTR of Sp1 (top). Mutations in the 3'UTR of Sp1 (bottom) are shown. (B) miR-375 inhibited the luciferase reporter activity of the wild-type 3'UTR of Sp1, but not the mutated 3'UTR of Sp1. SCC-15 and CAL27 cells were co-transfected with firefly luciferase reporter plasmid containing the wild-type or mutant 3'UTR of Sp1, as well as pRL-TK (a plasmid expressing Renilla luciferase) and the miR-375 mimic or the miRNA mimic control (NC) as indicated. After $24 \mathrm{~h}$, firefly luciferase activity was measured and normalized to Renilla luciferase activity. (C) Inhibition of Sp1 mRNA expression by miR-375 overexpression. SCC-15 and CAL27 cells were transfected with the miR-375 mimic or the miRNA mimic control (NC) as indicated. The mRNA expression of Sp1 was quantified by qRT-PCR $24 \mathrm{~h}$ after transfection. (D) Inhibition of Sp1 protein expression by miR-375 overexpression. SCC-15 and CAL27 cells were transfected as in C. After 48 h, Sp1 and $\beta$-actin (internal control) were detected by western blotting. Data are shown as the mean $\pm \mathrm{SD}\left({ }^{* *} \mathrm{P}<0.01\right)$.

cervical cancer (19). Since Sp1 is an important transcription factor in cellular processes (32-34), it is reasonable that the downregulation of Sp1 contributes to the inhibition of TSCC cell growth.

We further demonstrated that overexpression of miR-375 in TSCC cells downregulated cyclin D1 via Sp1, leading to cell cycle arrest (Fig. 7). Cyclin D1 is a key protein involved in cell cycle control and is essential for $\mathrm{G}_{1}$ to $\mathrm{S}$ transition $(35,36)$, and is regulated by Sp1 (20). More importantly, several studies have shown that the dysregulation of cyclin D1 contributes to HNSCC development (37-39) and our recent study also demonstrated that cyclin D1 plays an important role in cell growth and cell cycle progression in TSCC cell lines (8). Therefore, the fact that miR-375 inhibited cell proliferation and blocked $\mathrm{G}_{1}$ to $\mathrm{S}$ transition can at least partially be explained by the Sp1-dependent downregulation of cyclin D1.

However, as a ubiquitous transcription factor, $\mathrm{Sp} 1$ is also reported to regulate cell cycle progression by interaction with 
A

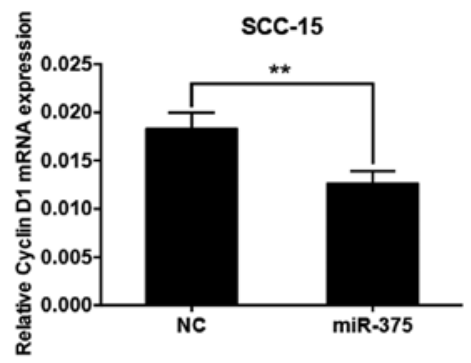

B

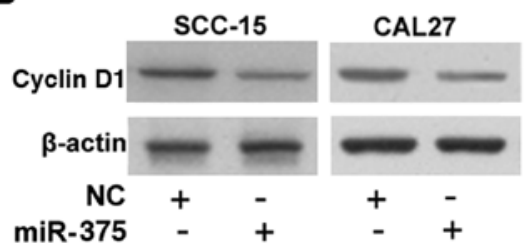

C

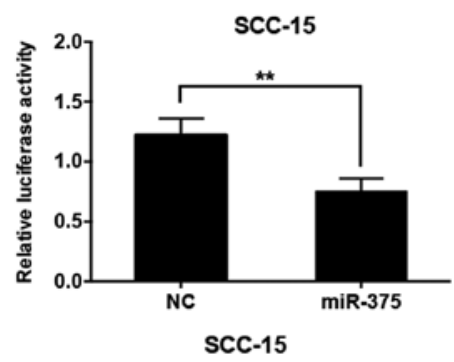

D

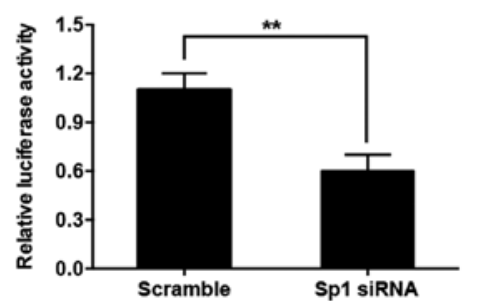

E

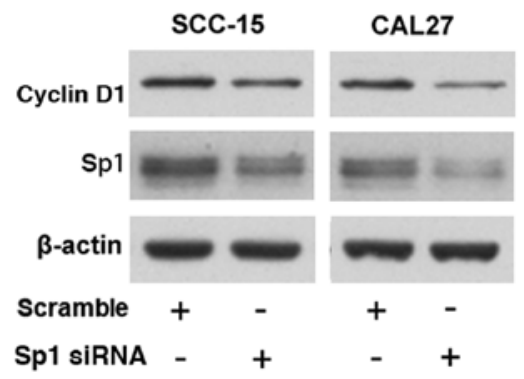

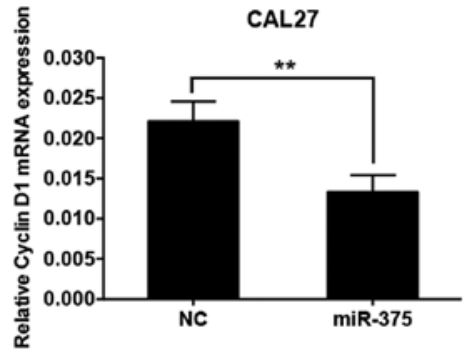
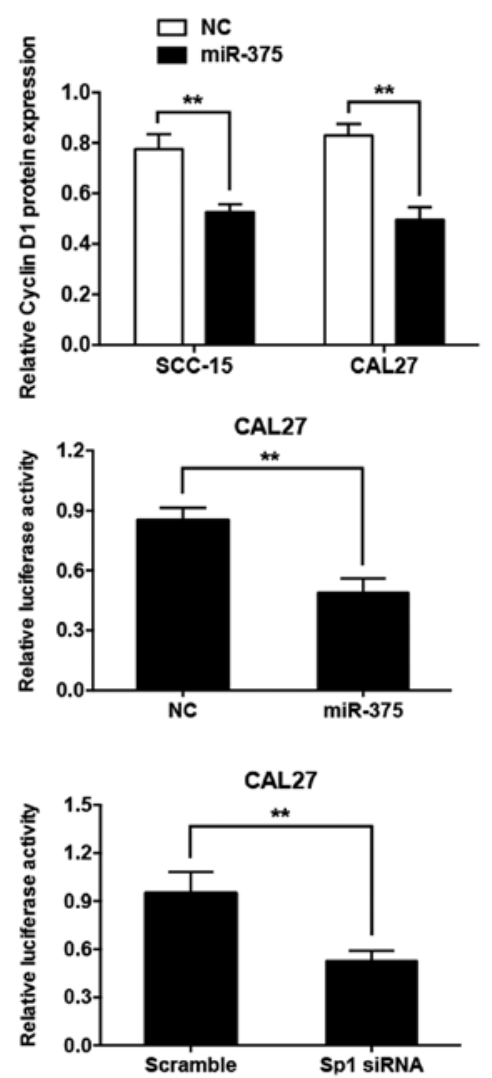

scC-15
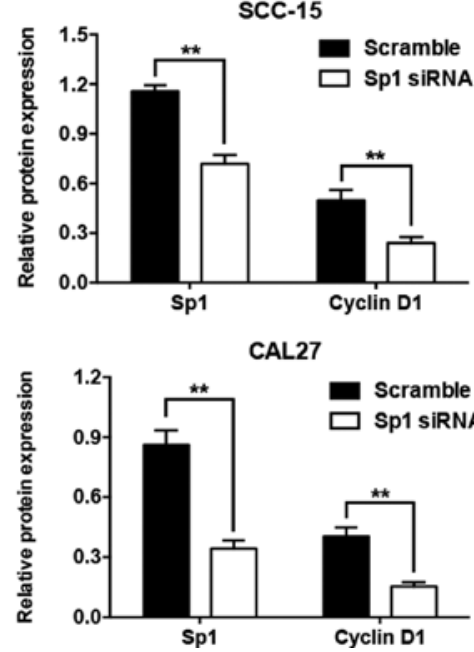

Figure 5. miR-375 downregulates cyclin D1 expression by reducing Sp1 expression. (A) Downregulation of cyclin D1 mRNA expression by miR-375 overexpression. SCC-15 and CAL27 cells were transfected with the miR-375 mimic or the miRNA mimic control (NC). The mRNA expression of cyclin D1 was quantified by qRT-PCR $24 \mathrm{~h}$ after transfection. (B) Downregulation of cyclin D1 protein expression by miR-375 overexpression. SCC-15 and CAL27 cells were transfected as in A. After $48 \mathrm{~h}$, cyclin D1 and $\beta$-actin (internal control) were detected by western blotting. (C) miR-375 increased the cyclin D1 promoter luciferase reporter activity. SCC-15 and CAL27 cells were co-transfected with a firefly luciferase reporter plasmid containing the cyclin D1 promoter, the pRL-TK plasmid, and the miR-375 mimic or the miRNA mimic control (NC). After $24 \mathrm{~h}$, firefly luciferase activity was measured and normalized to Renilla luciferase activity. (D) Sp1 knockdown upregulated cyclin D1 promoter luciferase reporter activity. SCC-15 and CAL27 cells were co-transfected with the firefly luciferase reporter plasmid containing the cyclin D1 promoter, pRL-TK, and siRNA control (scramble) or Sp1 siRNA. After 24 h, firefly luciferase activity was measured and normalized to Renilla luciferase activity. (E) Expression analysis of cyclin D1 after Sp1 knockdown. SCC-15 and CAL27 cells were transfected with siRNA control (scramble) or Sp1 siRNA. After 48 h, Sp1, cyclin D1 and $\beta$-actin (internal control) were detected by western blotting. Data are shown as the mean $\pm \mathrm{SD}\left({ }^{* * *} \mathrm{P}<0.01\right)$. 
A

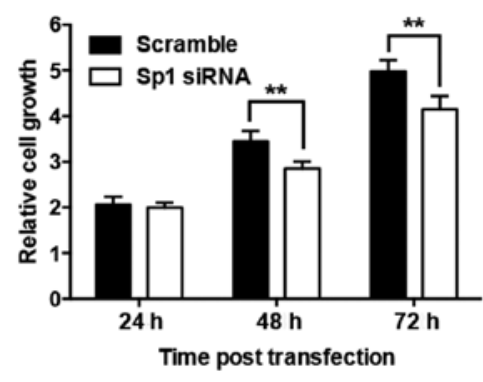

B

B NC Sp1 siRNA

SCC-15
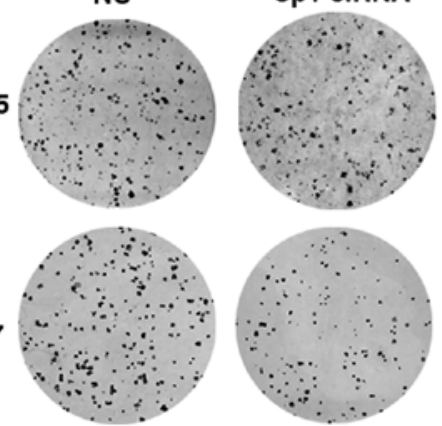
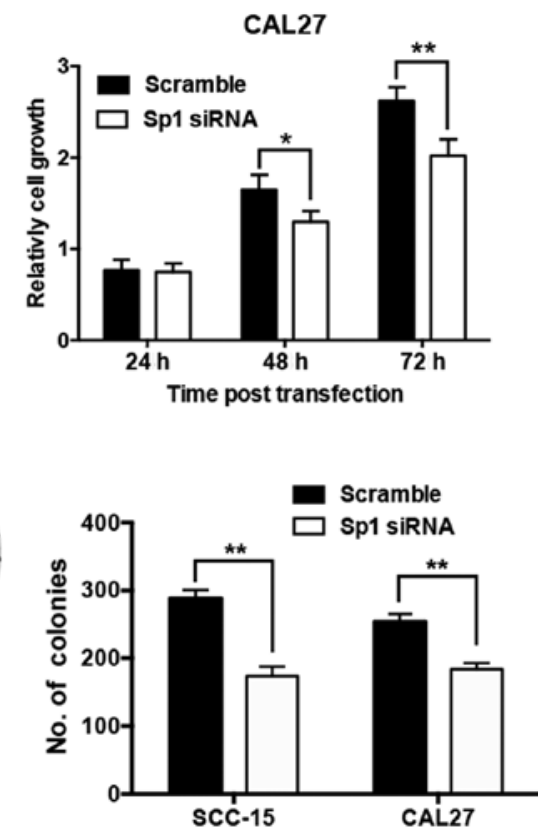

C $\mathrm{scC}-15$

NC

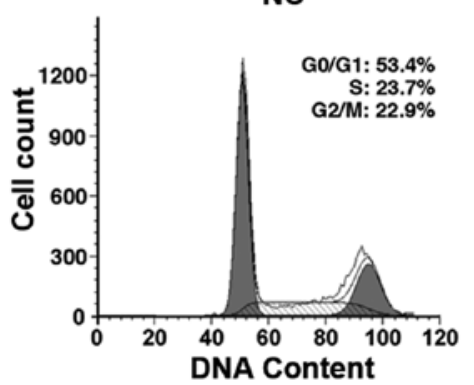

miR-375

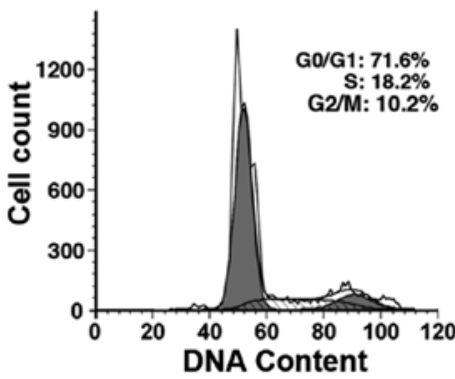

\section{CAL27}
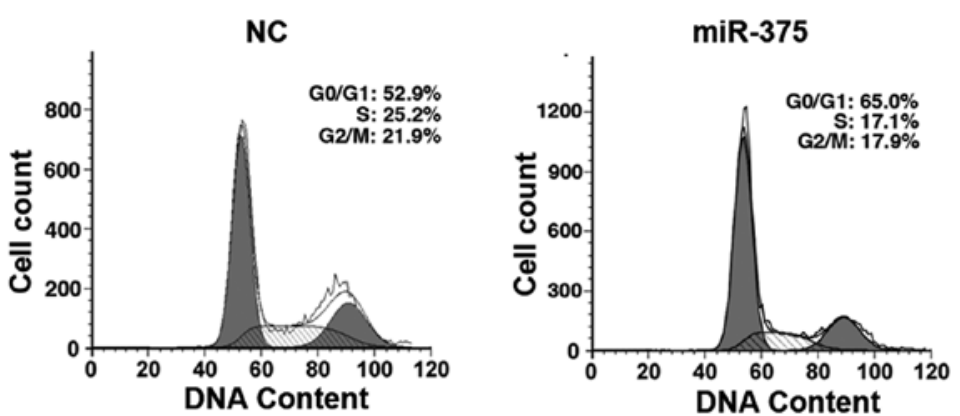

SCC-15
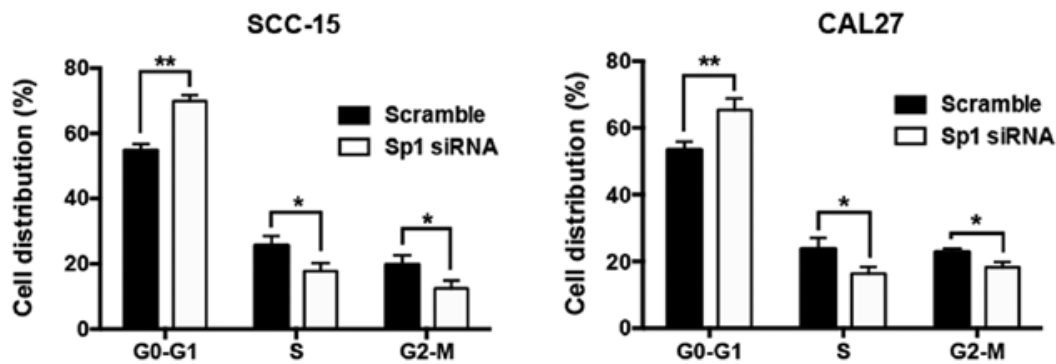

Figure 6. Sp1 knockdown mimics the effects of miR-375 overexpression. (A) Sp1 knockdown suppressed cell growth. SCC-15 and CAL27 cells were transfected with the siRNA control (scramble) or Sp1 siRNA. Cell proliferation was measured by the CCK-8 assay. (B) Sp1 knockdown inhibited colony formation. Representative results of the colony formation assay in SCC-15 and CAL27 cells transfected as in A are shown. (C) Cell cycle arrest due to Sp1 knockdown. SCC-15 and CAL27 cells were transfected as in A. Cells were stained with propidium iodide (PI) at $48 \mathrm{~h}$ post-transfection and analyzed by FACS. Data are shown as the mean $\pm \mathrm{SD}\left({ }^{*} \mathrm{P}<0.05,{ }^{* *} \mathrm{P}<0.01\right)$ 


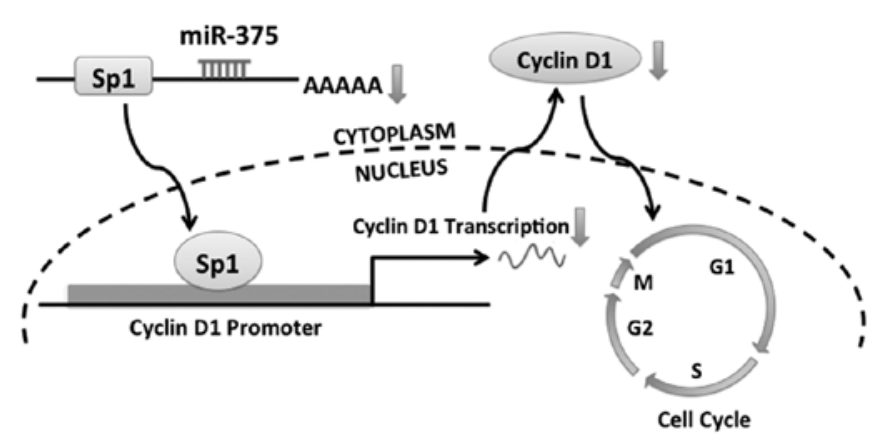

Figure 7. The steps involved in the tumor-suppressor role of miR-375 in TSCC cells. miR-375 targets the Sp1 transcript, resulting in inhibition of Sp1 and consequent downregulation of cyclin D1, leading to cell cycle arrest.

many other genes, including those encoding retinoblastoma protein $(40,41)$, retinoblastoma-related protein p107 (42), the transcription factor E2F (43), p53 (44) and mdm2 (45). We cannot exclude the possibility that miR-375 inhibits TSCC growth by simultaneously regulating other cell cycle regulatory proteins via Sp1. Further study is needed to identify other possible signaling pathways downstream of miR-375 in the suppression of TSCC growth.

In conclusion, miR-375 expression was reduced in TSCC and its downregulation was correlated with a poor prognosis in TSCC patients. Overexpression of miR-375 in TSCC cells inhibited proliferation and induced cell cycle arrest through an Sp1-cyclin D1 signaling pathway. These results suggest that miR-375 inhibits cell growth and is correlated with clinical outcomes in TSCC.

\section{Acknowledgements}

This study was supported by the National Natural Science Foundation of China (81402235,81472527), and the Foundation of the Peking University School and Hospital of Stomatology (PKUSS20140104).

\section{References}

1. Leemans CR, Braakhuis BJ and Brakenhoff RH: The molecular biology of head and neck cancer. Nat Rev Cancer 11: 9-22, 2011.

2. Greenlee RT, Hill-Harmon MB, Murray T and Thun M: Cancer statistics, 2001. CA Cancer J Clin 51: 15-36, 2001.

3. Patel SG and Shah JP: TNM staging of cancers of the head and neck: striving for uniformity among diversity. CA Cancer J Clin 55: 242-258, 2005.

4. Iorio MV and Croce CM: MicroRNA dysregulation in cancer: diagnostics, monitoring and therapeutics. A comprehensive review. EMBO Mol Med 4: 143-159, 2012.

5. Shah NG, Trivedi TI, Tankshali RA, et al: Prognostic significance of molecular markers in oral squamous cell carcinoma: a multivariate analysis. Head Neck 31: 1544-1556, 2009.

6. Inui M, Martello G and Piccolo S: MicroRNA control of signal transduction. Nat Rev Mol Cell Biol 11: 252-263, 2010.

7. Bartels CL and Tsongalis GJ: MicroRNAs: novel biomarkers for human cancer. Clin Chem 55: 623-631, 2009.

8. Jia LF, Wei SB, Gong K, Gan YH and Yu GY: Prognostic implications of micoRNA miR-195 expression in human tongue squamous cell carcinoma. PLoS One 8: e56634, 2013.

9. Li J, Huang H, Sun L, et al: MiR-21 indicates poor prognosis in tongue squamous cell carcinomas as an apoptosis inhibitor. Clin Cancer Res 15: 3998-4008, 2009.
10. Sun L, Yao Y, Liu B, et al: MiR-200b and miR-15b regulate chemotherapy-induced epithelial-mesenchymal transition in human tongue cancer cells by targeting BMI1. Oncogene 31: 432-445, 2012.

11. Siow M, Ng LK, Chong VV, et al: Dysregulation of miR-31 and miR-375 expression is associated with clinical outcomes in oral carcinoma. Oral Dis 20: 345-351, 2014.

12. Harris T, Jimenez L, Kawachi N, et al: Low-level expression of miR-375 correlates with poor outcome and metastasis while altering the invasive properties of head and neck squamous cell carcinomas. Am J Pathol 180: 917-928, 2012.

13. Bell RB, Kademani D, Homer L, Dierks EJ and Potter BE: Tongue cancer: Is there a difference in survival compared with other subsites in the oral cavity. J Oral Maxillofac Surg 65: 229-236, 2007.

14. Shaw RJ, McGlashan G, Woolgar JA, et al: Prognostic importance of site in squamous cell carcinoma of the buccal mucosa. Br J Oral Maxillofac Surg 47: 356-359, 2009.

15. Ding L, Xu Y,Zhang W, et al: MiR-375 frequently downregulated in gastric cancer inhibits cell proliferation by targeting JAK2. Cell Res 20: 784-793, 2010.

16. Zhou J, Song S, He S, et al: MicroRNA-375 targets PDK1 in pancreatic carcinoma and suppresses cell growth through the Akt signaling pathway. Int J Mol Med 33: 950-956, 2014.

17. Kong KL, Kwong DL, Chan TH, et al: MicroRNA-375 inhibits tumour growth and metastasis in oesophageal squamous cell carcinoma through repressing insulin-like growth factor 1 receptor. Gut 61: 33-42, 2012.

18. Zhao H, Zhu L, Jin Y, Ji H, Yan X and Zhu X: miR-375 is highly expressed and possibly transactivated by achaete-scute complex homolog 1 in small-cell lung cancer cells. Acta Biochim Biophys Sin 44: 177-182, 2012.

19. Wang F, Li Y, Zhou J, et al: miR-375 is down-regulated in squamous cervical cancer and inhibits cell migration and invasion via targeting transcription factor SP1. Am J Pathol 179: 2580-2588, 2011

20. Nagata D, Suzuki E, Nishimatsu H, et al: Transcriptional activation of the cyclin D1 gene is mediated by multiple cis-elements, including SP1 sites and a cAMP-responsive element in vascular endothelial cells. J Biol Chem 276: 662-669, 2001.

21. Grinstein E, Jundt F, Weinert I, Wernet P and Royer HD: Sp1 as G1 cell cycle phase specific transcription factor in epithelial cells. Oncogene 21: 1485-1492, 2002.

22. Lu Y, Thomson JM, Wong HY, Hammond SM and Hogan BL: Transgenic over-expression of the microRNA miR-17-92 cluster promotes proliferation and inhibits differentiation of lung epithelial progenitor cells. Dev Biol 310: 442-453, 2007.

23. Jia LF, Wei SB, Gan YH, et al: Expression, regulation and roles of miR-26a and MEG3 in tongue squamous cell carcinoma. Int J Cancer 135: 2282-2293, 2014.

24. Zhang WH, Gui JH, Wang CZ, et al: The identification of miR-375 as a potential biomarker in distal gastric adenocarcinoma. Oncol Res 20: 139-147, 2012.

25. Li J, Li X, Li Y, et al: Cell-specific detection of miR-375 downregulation for predicting the prognosis of esophageal squamous cell carcinoma by miRNA in situ hybridization. PLoS One 8: e53582, 2013.

26. Li Y, Jiang Q, Xia N, Yang H and Hu C: Decreased expression of microRNA-375 in nonsmall cell lung cancer and its clinical significance. J Int Med Res 40: 1662-1669, 2012.

27. Yu H, Jiang L, Sun C, et al: Decreased circulating miR-375: a potential biomarker for patients with non-small-cell lung cancer. Gene 534: 60-65, 2014.

28. Chang C, Shi H, Wang C, et al: Correlation of microRNA-375 downregulation with unfavorable clinical outcome of patients with glioma. Neurosci Lett 531: 204-208, 2012.

29. Wang Z, Hong Z, Gao F and Feng W: Upregulation of microRNA-375 is associated with poor prognosis in pediatric acute myeloid leukemia. Mol Cell Biochem 383: 59-65, 2013.

30. Li H, Shang H, Shu D, et al: gga-miR-375 plays a key role in tumorigenesis post subgroup $\mathrm{J}$ avian leukosis virus infection. PLoS One 9: e90878, 2014.

31. Wang Y, Tang Q, Li M, Jiang S and Wang X: MicroRNA-375 inhibits colorectal cancer growth by targeting PIK3CA. Biochem Biophys Res Commun 444: 199-204, 2014.

32. Cawley S, Bekiranov S, Ng HH, et al: Unbiased mapping of transcription factor binding sites along human chromosomes 21 and 22 points to widespread regulation of noncoding RNAs. Cell 116: 499-509, 2004. 
33. Michinaga S, Ishida A, Takeuchi R and Koyama Y: Endothelin-1 stimulates cyclin D1 expression in rat cultured astrocytes via activation of Sp1. Neurochem Int 63: 25-34, 2013.

34. Cram EJ, Liu BD, Bjeldanes LF and Firestone GL: Indole-3carbinol inhibits CDK6 expression in human MCF-7 breast cancer cells by disrupting Sp1 transcription factor interactions with a composite element in the CDK6 gene promoter. J Biol Chem 276: 22332-22340, 2001.

35. Baldin V, Lukas J, Marcote MJ, Pagano M and Draetta G: Cyclin $\mathrm{D} 1$ is a nuclear protein required for cell cycle progression in G1. Genes Dev 7: 812-821, 1993.

36. Motokura T, Keyomarsi K, Kronenberg HM and Arnold A: Cloning and characterization of human cyclin D3, a cDNA closely related in sequence to the PRAD1/cyclin D1 protooncogene. J Biol Chem 267: 20412-20415, 1992.

37. Bova RJ, Quinn DI, Nankervis JS, et al: Cyclin D1 and p16INK4A expression predict reduced survival in carcinoma of the anterior tongue. Clin Cancer Res 5: 2810-2819, 1999.

38. Michalides R, van Veelen N, Hart A, Loftus B, Wientjens E and Balm A: Overexpression of cyclin D1 correlates with recurrence in a group of forty-seven operable squamous cell carcinomas of the head and neck. Cancer Res 55: 975-978, 1995.

39. Huang SF, Cheng SD, Chuang WY, et al: Cyclin D1 overexpression and poor clinical outcomes in Taiwanese oral cavity squamous cell carcinoma. World J Surg Oncol 10: 40, 2012.
40. Chang YC, Illenye S and Heintz NH: Cooperation of E2F-p130 and Sp1-pRb complexes in repression of the Chinese hamster dhfr gene. Mol Cell Biol 21: 1121-1131, 2001.

41. Kim SJ, Onwuta US, Lee YI, Li R, Botchan MR and Robbins PD The retinoblastoma gene product regulates Sp1-mediated transcription. Mol Cell Biol 12: 2455-2463, 1992.

42. Datta PK, Raychaudhuri P and Bagchi S: Association of p107 with Sp1: genetically separable regions of p107 are involved in regulation of E2F- and Sp1-dependent transcription. Mol Cell Biol 15: 5444-5452, 1995.

43. Karlseder J, Rotheneder H and Wintersberger E: Interaction of Sp1 with the growth- and cell cycle-regulated transcription factor E2F. Mol Cell Biol 16: 1659-1667, 1996.

44. Gualberto A and Baldwin AS Jr: p53 and Sp1 interact and cooperate in the tumor necrosis factor-induced transcriptional activation of the HIV-1 long terminal repeat. J Biol Chem 270: 19680-19683, 1995.

45. Johnson-Pais T, Degnin C and Thayer MJ: pRB induces Sp1 activity by relieving inhibition mediated by MDM2. Proc Natl Acad Sci USA 98: 2211-2216, 2001. 